\title{
Mathematical Theory of Normal Waves in an Open Metal-Dielectric Regular Waveguide of Arbitrary Cross Section
}

\section{Eugene Smolkin ${ }^{a, b}$ and Yury Smirnov Sm $^{a, b}$}

${ }^{a}$ Penza State University

Department of Mathematics and Supercomputing, Penza State University, Krasnaya Str. 40, 440026 Penza, Russia

${ }^{b}$ Sirius University of Science and Technology

1 Olympic Ave, 354340 Sochi, Russia

E-mail(corresp.): e.g.smolkin@hotmail.com

E-mail: smirnovyug@mail.ru

Received July 5, 2019; revised March 28, 2020; accepted April 7, 2020

\begin{abstract}
The problem of normal waves in an open metal-dielectric regular waveguide of arbitrary cross-section is considered. This problem is reduced to the boundary eigenvalue problem for longitudinal components of electromagnetic field in Sobolev spaces. To find the solution, we use the variational formulation of the problem. The variational problem is reduced to study of an operator-function. Discreteness of the spectrum is proved and distribution of the characteristic numbers of the operatorfunction on the complex plane is found.
\end{abstract}

Keywords: non-linear eigenvalue problem, Maxwell's equations, operator-function, Sobolev spaces, discrete spectrum.

AMS Subject Classification: 34B07; 47A10; 78A50.

\section{Introduction}

Analysis of wave propagation in open metal-dielectric waveguides constitutes an important class of vector electromagnetic problems. An open waveguide with an arbitrary cross section is studied in the paper.

The typical problems studied here are non self-adjoint boundary eigenvalue problems for the systems of Helmholtz equations with piecewise constant coefficients and transmission conditions containing the spectral parameter. On

Copyright (c) 2020 The Author(s). Published by VGTU Press

This is an Open Access article distributed under the terms of the Creative Commons Attribution License (http://creativecommons.org/licenses/by/4.0/), which permits unrestricted use, distribution, and reproduction in any medium, provided the original author and source are credited. 
the discontinuity lines (surfaces) additional conditions called transmission conditions should be satisfied. However, the spectral parameter appears only usually in the equations and does not enter into transmission conditions, and so we have an eigenvalue problem for the usually self-adjoint operator. Sometimes, however, the spectral parameter occurs not only in the equation, but also in the transmission conditions, and often nonlinearly. We thus obtain a non self-adjoint problem.

An approach based on the reduction to eigenvalue problems for operator pencils considered in Sobolev spaces was proposed by Smirnov in $[17,18,19]$ (see also $[14,15])$. The general theory of polynomial operator-functions called operator pencils is sufficiently well elaborated. A fundamental work by Keldysh [9] pioneered investigation of non-self-adjoint polynomial pencils.

The method of operator pencils is known to be a natural and efficient approach for investigation of the wave propagation in regular waveguides. Operator pencils were applied to the analysis of electromagnetic problems in $[4,11,30]$.

Open waveguide structures were investigated by a number of authors $[4$, $12,13]$. However, for open (unshielded) structures, a complete theory of wave propagation is not constructed. In this case the problem becomes much more complicated (due to the non-compactness of the corresponding operators). This article deals with open structures i.e. the case of an unbounded exterior domain is considered. The first results on the investigation of such problems were recently obtained in $[20,23,24,25]$ for a circular waveguide.

These difficulties can be overcome by introducing a fictitious outer region (the exterior of the circle) and representing the solution in this region in terms of the Green's function. This leads to the appearance of a trace operator (on the boundary of the fictitious region) in the variational relation, which depends in a nonlinear way on the spectral parameter. We have to analyze not the operator pencil (as in [19]), but an operator-function. Nevertheless, it is possible to study the properties of the operator-function in sufficient detail and obtain results on its spectrum. The discreteness of the spectrum of the problem of normal waves is proved in the article. The results on the distribution of characteristic numbers on the complex plane are presented.

Note that we consider waves that decrease at a distance from the waveguide (we impose the corresponding conditions at infinity). Other types of waves are not considered. This approach was used to study the shielded waveguide structures as well $[21,22]$.

\section{Statement of the problem}

Consider the three-dimensional space $\mathbb{R}^{3}$ with the cylindrical coordinate system $O \rho \varphi z$. The space is filled with an isotropic source-free medium with permittivity $\varepsilon_{2} \varepsilon_{0} \equiv$ const, $\varepsilon_{2}>1$ and permeability $\mu_{0} \equiv$ const, where $\varepsilon_{0}$ and $\mu_{0}$ are permittivity and permeability of vacuum.

We will consider a mathematical model of a regular (along the $O z$ axis), open waveguide structure, whose transverse section by the plane $z=$ const is formed by the bounded domain $\Omega_{1}$. The boundary $\Gamma_{0}$ is the cross-section of the surface of the infinitely thin and perfectly conducting screens and $\Gamma_{1}$ 


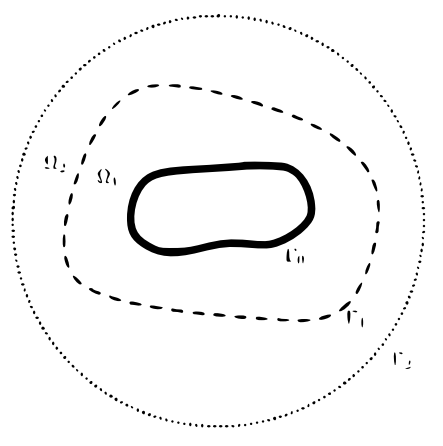

Figure 1. Geometry of the problem.

is the cross-section of the dielectric surfaces. The waveguide is filled with a homogeneous isotropic dielectric with a relative permittivity $\varepsilon>\varepsilon_{0}, \operatorname{Im} \varepsilon=$ $0, \mu=\mu_{0}$.

We choose $r>0$ so that $B_{r}:=\{x=(\rho, \varphi): \rho<r\} \supset \bar{\Omega}_{1}$. We introduce the domain $\Omega_{2}:=B_{r} \backslash \bar{Q} ; \Gamma_{2}:=\{x: \rho=r\}$ (Figure 1).

The dielectric permittivity in the whole space is given by the following expression $\varepsilon_{0} \widetilde{\varepsilon}$, where

$$
\widetilde{\varepsilon}=\left\{\begin{array}{lr}
\varepsilon_{1}, & x \in \Omega_{1}, \\
\varepsilon_{2}, & x \in \Omega_{2}, \\
\varepsilon_{2}, & x \in \mathbb{R}^{2} \backslash \bar{\Omega}_{1} .
\end{array}\right.
$$

We will consider monochromatic waves

$$
\mathbf{E} e^{-i \omega t}=e^{-i \omega t}\left(\mathrm{E}_{\rho}, \mathrm{E}_{\varphi}, \mathrm{E}_{z}\right)^{T}, \mathbf{H} e^{-i \omega t}=e^{-i \omega t}\left(\mathrm{H}_{\rho}, \mathrm{H}_{\varphi}, \mathrm{H}_{z}\right)^{T},
$$

where $(\cdot)^{T}$ denotes the transpose operation. Each component of the field $\mathbf{E}$, $\mathbf{H}$ is a function of three spatial variables.

The problem of normal waves in a waveguide structure is the problem of finding nontrivial propagating wave solutions of the homogeneous system of Maxwell equations, i.e., solutions with dependence of the from $e^{i \gamma z}$ on the coordinate $z[26]$,

$$
\begin{gathered}
\left\{\begin{array}{l}
\operatorname{rot} \mathbf{H}=-i \tilde{\varepsilon} \mathbf{E}, \\
\operatorname{rot} \mathbf{E}=i \mathbf{H},
\end{array}\right. \\
\mathbf{E}=\left(E_{\rho}(x) \mathbf{e}_{\rho}+E_{\varphi}(x) \mathbf{e}_{\varphi}+E_{z}(x) \mathbf{e}_{z}\right) e^{i \gamma z}, \\
\mathbf{H}=\left(H_{\rho}(x) \mathbf{e}_{\rho}+H_{\varphi}(x) \mathbf{e}_{\varphi}+H_{z}(x) \mathbf{e}_{z}\right) e^{i \gamma z},
\end{gathered}
$$

with boundary conditions for tangential electric components on perfectly conducting surfaces,

$$
\left.E_{\tau}\right|_{\Gamma_{0}}=0,
$$

transmission conditions for tangential electric and magnetic components on surfaces of "jump" of permittivity,

$$
\begin{array}{ll}
{\left.\left[E_{\tau}\right]\right|_{\Gamma_{1}}=0,} & {\left.\left[H_{\tau}\right]\right|_{\Gamma_{1}}=0} \\
{\left.\left[E_{\tau}\right]\right|_{\Gamma_{2}}=0,} & {\left.\left[H_{\tau}\right]\right|_{\Gamma_{2}}=0,}
\end{array}
$$


the finite energy condition

$$
\int_{V}\left(\widetilde{\varepsilon}|\mathbf{E}|^{2}+|\mathbf{H}|^{2}\right) d X<\infty, V=\left\{X: x \in \Omega_{1} \cup \Omega_{2}\right\}, X=(\rho, \varphi, z)
$$

and the radiation condition at infinity: the electromagnetic field decays as $O(1 / \rho)$ for $\rho \rightarrow \infty$.

Here $\gamma$ is the normalized propagation constant of the waveguide (unknown spectral parameter of the problem). The problem (2.1)-(2.3) is an eigenvalue problem for the Maxwell equations with spectral parameter $\gamma$. In what follows we often omit the arguments of functions when it does not lead to misunderstanding.

The Maxwell system (2.1) is written in the normalized form. The passage to dimensionless variables has been carried out [19]; namely, $k_{0} \rho \rightarrow \rho, \gamma \rightarrow \frac{\gamma}{k_{0}}$, $\sqrt{\frac{\mu_{0}}{\varepsilon_{0}}} \mathbf{H} \rightarrow \mathbf{H}, \mathbf{E} \rightarrow \mathbf{E}$, where $k_{0}^{2}=\omega^{2} \varepsilon_{0} \mu_{0}$. The time factor $e^{-i \omega t}$ is omitted everywhere. Substituting $\mathbf{E}$ and $\mathbf{H}$ with components (2.2) into equations (2.1), we obtain

$$
\left\{\begin{aligned}
\frac{1}{\rho} \frac{\partial H_{z}}{\partial \varphi}-i \gamma H_{\varphi} & =-i \widetilde{\varepsilon} E_{\rho}, \\
i \gamma H_{\rho}-\frac{\partial H_{z}}{\partial \rho} & =-i \widetilde{\varepsilon} E_{\varphi}, \\
\frac{1}{\rho} \frac{\partial\left(\rho H_{\varphi}\right)}{\partial \rho}-\frac{1}{\rho} \frac{\partial H_{\rho}}{\partial \varphi} & =-i \widetilde{\varepsilon} E_{z}, \\
\frac{1}{\rho} \frac{\partial E_{z}}{\partial \varphi}-i \gamma E_{\varphi} & =i H_{\rho}, \\
i \gamma E_{\rho}-\frac{\partial E_{z}}{\partial \rho} & =i H_{\varphi}, \\
\frac{1}{\rho} \frac{\partial\left(\rho E_{\varphi}\right)}{\partial \rho}-\frac{1}{\rho} \frac{\partial E_{\rho}}{\partial \varphi} & =i H_{z},
\end{aligned}\right.
$$

Expressing the functions $E_{\rho}, H_{\rho}, E_{\varphi}, H_{\varphi}$ through $E_{z}$ and $H_{z}$ from the 1st, 2nd, 4 th and 5 th equation of system (2.4), we find

$$
\begin{aligned}
E_{\rho}=\frac{1}{\rho} \frac{i}{\widetilde{\kappa}^{2}}\left(\gamma \rho \frac{\partial E_{z}}{\partial \rho}+\frac{\partial H_{z}}{\partial \varphi}\right), & H_{\rho}=\frac{1}{\rho} \frac{i}{\widetilde{\kappa}^{2}}\left(\gamma \rho \frac{\partial H_{z}}{\partial \rho}-\widetilde{\varepsilon} \frac{\partial E_{z}}{\partial \varphi}\right), \\
E_{\varphi}=\frac{1}{\rho} \frac{i}{\widetilde{\kappa}^{2}}\left(\gamma \frac{\partial E_{z}}{\partial \varphi}-\rho \frac{\partial H_{z}}{\partial \rho}\right), & H_{\varphi}=\frac{1}{\rho} \frac{i}{\widetilde{\kappa}^{2}}\left(\gamma \frac{\partial H_{z}}{\partial \varphi}+\rho \widetilde{\varepsilon} \frac{\partial E_{z}}{\partial \rho}\right),
\end{aligned}
$$

where $\widetilde{\kappa}^{2}=\widetilde{\varepsilon}-\gamma^{2}$. It follows from (2.5) that the field of the normal wave can be represented via two scalar functions $\Pi:=E_{z}(x), \Phi:=H_{z}(x)$.

For functions $\Pi$ and $\Phi$ from (2.1)-(2.3) we have the following eigenvalue problem: to find $\gamma \in \mathbb{C}$ (called eigenvalues) such that there are nontrivial solutions of the system

$$
\begin{aligned}
\Delta \Pi+\widetilde{\kappa}^{2} \Pi & =0, \\
\Delta \Phi+\widetilde{\kappa}^{2} \Phi & =0,
\end{aligned}
$$


satisfying the boundary conditions on $\Gamma_{0}$,

$$
\left.\Pi\right|_{\Gamma_{0}}=0,\left.\frac{\partial \Phi}{\partial n}\right|_{\Gamma_{0}}=0
$$

the transmission conditions on $\Gamma_{1}$,

$$
\begin{gathered}
{\left.[\Pi]\right|_{\Gamma_{1}}=0,\left.\quad[\Phi]\right|_{\Gamma_{1}}=0} \\
\left.\gamma\left[\frac{1}{\widetilde{\kappa}^{2}} \frac{\partial \Pi}{\partial \tau}\right]\right|_{\Gamma_{1}}-\left.\left[\frac{1}{\widetilde{\kappa}^{2}} \frac{\partial \Phi}{\partial n}\right]\right|_{\Gamma_{1}}=0,\left.\quad \gamma\left[\frac{1}{\widetilde{\kappa}^{2}} \frac{\partial \Phi}{\partial \tau}\right]\right|_{\Gamma_{1}}+\left.\left[\frac{\widetilde{\varepsilon}}{\widetilde{\kappa}^{2}} \frac{\partial \Pi}{\partial n}\right]\right|_{\Gamma_{1}}=0
\end{gathered}
$$

the transmission conditions on $\Gamma_{2}$,

$$
\begin{aligned}
& {\left.[\Pi]\right|_{\Gamma_{2}}=0,\left.[\Phi]\right|_{\Gamma_{2}}=0} \\
& {\left.\left[\frac{\partial \Pi}{\partial n}\right]\right|_{\Gamma_{2}}=0,\left.\left[\frac{\partial \Phi}{\partial n}\right]\right|_{\Gamma_{2}}=0}
\end{aligned}
$$

where $n$ denotes the normal unit vector such that $\rho \times \varphi=\tau \times n$, the energy condition

$$
\int_{\Omega}\left(|\nabla \Pi|^{2}+|\nabla \Phi|^{2}+|\Pi|^{2}+|\Phi|^{2}\right) d x<\infty
$$

where $\Omega=\Omega_{1} \cup \Omega_{2} \cup \Gamma_{1}$, and radiation condition at infinity

$$
\Pi(\rho, \varphi)=O(1 / \rho), \Phi(\rho, \varphi)=O(1 / \rho), \quad \rho \rightarrow \infty \text {, uniformly w.r.t } \varphi \text {. }
$$

Once we determine the longitudinal field components $\Pi$ and $\Phi$, we can find the transverse components by formulas (2.3). The equivalence of reduction to the problem $(2.6)-(2.11)$ is not valid only for $\gamma^{2}=\widetilde{\varepsilon}$; in this case it is necessary to study the system (2.1) directly.

\section{Variational formulation}

We will find the solutions $\Pi$ and $\Phi$ of the problem (2.6)-(2.11) in Sobolev spaces: $H_{0}^{1}(\Omega)$ and $H^{1}(\Omega)$, respectively, with the inner product and the norm

$$
(f, g)_{1}=\int_{\Omega}(\nabla f \nabla \bar{g}+f \bar{g}) d x,\|f\|_{1}^{2}=(f, f)_{1} .
$$

Remark 1. Here we use the notation for the Sobolev space $H_{0}^{1}(\Omega)$, which does not coincide with the standard one: in our case $\left.f\right|_{\Gamma_{0}}=0$ but generally $\left.f\right|_{\Gamma_{2}} \neq 0$.

Let us give the variational formulation of the problem (2.6)-(2.11). Multiplying the equations of system (2.6) by arbitrary test functions $u \in H_{0}^{1}(\Omega), v \in$ $H^{1}(\Omega)$ (we can assume that these functions are continuously differentiable in $\bar{\Omega})$, and applying Green's formula [3], we obtain

$$
\int_{\Omega_{1}} \bar{u} \Delta \Pi d x+\int_{\Omega_{1}} \kappa_{1}^{2} \Pi \bar{u} d x=\int_{\Gamma_{1}} \frac{\partial \Pi}{\partial n} \bar{u} d \tau-\int_{\Gamma_{0}} \frac{\partial \Pi}{\partial n} \bar{u} d \tau-\int_{\Omega_{1}} \nabla \Pi \nabla \bar{u} d x+\int_{\Omega_{1}} \kappa_{1}^{2} \Pi \bar{u} d x=0,
$$


and

$\int_{\Omega_{1}} \bar{v} \Delta \Phi d x+\int_{\Omega_{1}} \kappa_{1}^{2} \Phi \bar{v} d x=\int_{\Gamma_{1}} \frac{\partial \Phi}{\partial n} \bar{v} d \tau-\int_{\Gamma_{0}} \frac{\partial \Phi}{\partial n} \bar{v} d \tau-\int_{\Omega_{1}} \nabla \Phi \nabla \bar{v} d x+\int_{\Omega_{1}} \kappa_{1}^{2} \Phi \bar{v} d x=0$ where $\kappa_{1}^{2}=\varepsilon_{1}-\gamma^{2}$. Taking into account the boundary conditions (2.7), we get

$$
\int_{\Omega_{1}} \bar{u} \Delta \Pi d x+\int_{\Omega_{1}} \kappa_{1}^{2} \Pi \bar{u} d x=\int_{\Gamma_{1}} \frac{\partial \Pi}{\partial n} \bar{u} d \tau-\int_{\Omega_{1}} \nabla \Pi \nabla \bar{u} d x+\int_{\Omega_{1}} \kappa_{1}^{2} \Pi \bar{u} d x=0,
$$

and

$$
\int_{\Omega_{1}} \bar{v} \Delta \Phi d x+\int_{\Omega_{1}} \kappa_{1}^{2} \Phi \bar{v} d x=\int_{\Gamma_{1}} \frac{\partial \Phi}{\partial n} \bar{v} d \tau-\int_{\Omega_{1}} \nabla \Phi \nabla \bar{v} d x+\int_{\Omega_{1}} \kappa_{1}^{2} \Phi \bar{v} d x=0 .
$$

Multiplying (3.1) and (3.2) by $\frac{\varepsilon_{1}}{\kappa_{1}^{2}}$ and $\frac{1}{\kappa_{1}^{2}}$, respectively; adding and subtracting $\frac{\gamma}{\kappa_{1}^{2}} \int_{\Gamma_{1}} \frac{\partial \Phi}{\partial \tau} \bar{u} d \tau$ and $\frac{\gamma}{\kappa_{1}^{2}} \int_{\Gamma_{1}} \frac{\partial \Pi}{\partial \tau} \bar{v} d \tau$, we get

$$
\begin{aligned}
\frac{\gamma}{\kappa_{1}^{2}} \int_{\Gamma_{1}} \frac{\partial \Phi}{\partial \tau} \bar{u} d \tau+\frac{\varepsilon_{1}}{\kappa_{1}^{2}} \int_{\Gamma_{1}} \frac{\partial \Pi}{\partial n} \bar{u} d \tau & =\frac{\gamma}{\kappa_{1}^{2}} \int_{\Gamma_{1}} \frac{\partial \Phi}{\partial \tau} \bar{u} d \tau+\frac{1}{\kappa_{1}^{2}} \int_{\Omega_{1}} \varepsilon_{1} \nabla \Pi \nabla \bar{u} d x-\int_{\Omega_{1}} \varepsilon_{1} \Pi \bar{u} d x \\
\frac{\gamma}{\kappa_{1}^{2}} \int_{\Gamma_{1}} \frac{\partial \Pi}{\partial \tau} \bar{v} & d \tau-\frac{1}{\kappa_{1}^{2}} \int_{\Gamma_{1}} \frac{\partial \Phi}{\partial n} \bar{v} d \tau \\
= & \frac{\gamma}{\kappa_{1}^{2}} \int_{\Gamma_{1}} \frac{\partial \Pi}{\partial \tau} \bar{v} d \tau-\frac{1}{\kappa_{1}^{2}} \int_{\Omega_{1}} \nabla \Phi \nabla \bar{v} d x+\int_{\Omega_{1}} \Phi \bar{v} d x
\end{aligned}
$$

For the domain $\Omega_{2}$, we obtain in a similar way

$$
\begin{aligned}
& -\frac{\gamma}{\kappa_{2}^{2}} \int_{\Gamma_{1}} \frac{\partial \Phi}{\partial \tau} \bar{u} d \tau-\frac{\varepsilon_{2}}{\kappa_{2}^{2}} \int_{\Gamma_{1}} \frac{\partial \Pi}{\partial n} \bar{u} d \tau \\
& =-\frac{1}{\kappa_{2}^{2}} \int_{\Gamma_{2}} \frac{\partial \Pi}{\partial n} \bar{u} d \tau-\frac{\gamma}{\kappa_{2}^{2}} \int_{\Gamma_{1}} \frac{\partial \Phi}{\partial \tau} \bar{u} d \tau+\frac{1}{\kappa_{2}^{2}} \int_{\Omega_{2}} \varepsilon_{2} \nabla \Pi \nabla \bar{u} d x+\int_{\Omega_{2}} \varepsilon_{2} \Pi \bar{u} d x \\
& -\frac{\gamma}{\kappa_{2}^{2}} \int_{\Gamma_{1}} \frac{\partial \Pi}{\partial \tau} \bar{v} d \tau+\frac{1}{\kappa_{2}^{2}} \int_{\Gamma_{1}} \frac{\partial \Phi}{\partial n} \bar{v} d \tau \\
& \quad=\frac{1}{\kappa_{2}^{2}} \int_{\Gamma_{2}} \frac{\partial \Phi}{\partial n} \bar{v} d \tau-\frac{\gamma}{\kappa_{2}^{2}} \int_{\Gamma_{1}} \frac{\partial \Pi}{\partial \tau} \bar{v} d \tau-\frac{1}{\kappa_{2}^{2}} \int_{\Omega_{2}} \nabla \Phi \nabla \bar{v} d x-\int_{\Omega_{2}} \Phi \bar{v} d x
\end{aligned}
$$

where $\kappa_{2}^{2}=\gamma^{2}-\varepsilon_{2}$.

Applying the transmission conditions (2.8) in the following form

$$
\begin{aligned}
& \frac{\gamma}{\kappa_{1}^{2}} \int_{\Gamma_{1}} \frac{\partial \Phi}{\partial \tau} \bar{u} d \tau+\frac{\varepsilon_{1}}{\kappa_{1}^{2}} \int_{\Gamma_{1}} \frac{\partial \Pi}{\partial n} \bar{u} d \tau=-\frac{\gamma}{\kappa_{2}^{2}} \int_{\Gamma_{1}} \frac{\partial \Phi}{\partial \tau} \bar{u} d \tau-\frac{\varepsilon_{2}}{\kappa_{2}^{2}} \int_{\Gamma_{1}} \frac{\partial \Pi}{\partial n} \bar{u} d \tau \\
& \frac{\gamma}{\kappa_{1}^{2}} \int_{\Gamma_{1}} \frac{\partial \Pi}{\partial \tau} \bar{v} d \tau-\frac{1}{\kappa_{1}^{2}} \int_{\Gamma_{1}} \frac{\partial \Phi}{\partial n} \bar{v} d \tau=-\frac{\gamma}{\kappa_{2}^{2}} \int_{\Gamma_{1}} \frac{\partial \Pi}{\partial \tau} \bar{v} d \tau+\frac{1}{\kappa_{2}^{2}} \int_{\Gamma_{1}} \frac{\partial \Phi}{\partial n} \bar{v} d \tau
\end{aligned}
$$


we have

$$
\begin{gathered}
\int_{\Gamma_{2}} \frac{\partial \Pi}{\partial n} \bar{u} d \tau=-\gamma \int_{\Gamma_{1}}\left(\frac{\kappa_{2}^{2}}{\kappa_{1}^{2}} \frac{\partial \Phi}{\partial \tau}+\frac{\partial \Phi}{\partial \tau}\right) \bar{u} d \tau-\kappa_{2}^{2} \int_{\Omega} \frac{\widetilde{\varepsilon}}{\tilde{k}^{2}} \nabla \Pi \nabla \bar{u} d x+\kappa_{2}^{2} \int_{\Omega} \widetilde{\varepsilon} \Pi \bar{u} d x, \\
-\int_{\Gamma_{2}} \frac{\partial \Phi}{\partial n} \bar{v} d \tau=-\gamma \int_{\Gamma_{1}}\left(\frac{\kappa_{2}^{2}}{\kappa_{1}^{2}} \frac{\partial \Pi}{\partial \tau}+\frac{\partial \Pi}{\partial \tau}\right) \bar{v} d \tau+\kappa_{2}^{2} \int_{\Omega} \frac{1}{\tilde{k}^{2}} \nabla \Phi \nabla \bar{v} d x-\kappa_{2}^{2} \int_{\Omega} \Phi \bar{v} d x .
\end{gathered}
$$

Since the boundary $\Gamma_{2}$ is a circle with radius $r$, we can rewrite the last formulas as follows

$$
\begin{aligned}
& \int_{\Gamma_{2}} \frac{\partial \Pi}{\partial \rho} \bar{u} d l=-\gamma \int_{\Gamma_{1}}\left(\frac{\kappa_{2}^{2}}{\kappa_{1}^{2}} \frac{\partial \Phi}{\partial \tau}+\frac{\partial \Phi}{\partial \tau}\right) \bar{u} d \tau-\kappa_{2}^{2} \int_{\Omega} \frac{\widetilde{\varepsilon}}{\tilde{k}^{2}} \nabla \Pi \nabla \bar{u} d x+\kappa_{2}^{2} \int_{\Omega} \widetilde{\varepsilon} \Pi \bar{u} d x \\
& \int_{\Gamma_{2}} \frac{\partial \Phi}{\partial \rho} \bar{v} d l=\gamma \int_{\Gamma_{1}}\left(\frac{\kappa_{2}^{2}}{\kappa_{1}^{2}} \frac{\partial \Pi}{\partial \tau}+\frac{\partial \Pi}{\partial \tau}\right) \bar{v} d \tau-\kappa_{2}^{2} \int_{\Omega} \frac{1}{\tilde{k}^{2}} \nabla \Phi \nabla \bar{v} d x+\kappa_{2}^{2} \int_{\Omega} \Phi \bar{v} d x .
\end{aligned}
$$

\subsection{The Green function}

Outside the domain $\Omega$ we have $\widetilde{\varepsilon}=\varepsilon_{2}$. Then the system (2.6) takes the form

$$
L \Pi:=\Delta \Pi-\kappa_{2}^{2} \Pi=0, \quad L \Phi:=\Delta \Phi-\kappa_{2}^{2} \Phi=0,
$$

where $\kappa_{2}^{2}=\gamma^{2}-\varepsilon_{2}$.

The Green's function $G$ of the exterior Dirichlet problem for the Helmholtz equation (3.3) in the exterior of the circle $\Gamma_{2}$ is defined as the solution of the following boundary-value problem:

$$
\left\{\begin{array}{l}
L G=-\frac{\delta\left(\rho-\rho_{0}\right) \delta\left(\varphi-\varphi_{0}\right)}{\rho}, \rho_{0}>r \\
\left.G\left(x, x_{0}\right)\right|_{\Gamma_{2}}=0 \\
G\left(x, x_{0}\right)=O(1 / \rho), \rho \rightarrow \infty .
\end{array}\right.
$$

We have $G\left(x, x_{0}\right)=G\left(x_{0}, x\right), x=(\rho, \varphi)$ and $x_{0}=\left(\rho_{0}, \varphi_{0}\right)$.

We have [10] in the sense of distributions

$$
\delta\left(\varphi-\varphi_{0}\right)=\frac{1}{2 \pi} \sum_{m=-\infty}^{\infty} \cos \left[m\left(\varphi-\varphi_{0}\right)\right] .
$$

Then the Green's function $G\left(x, x_{0}\right)$ takes the form

$$
G\left(x, x_{0}\right)=\frac{1}{2 \pi} \sum_{m=-\infty}^{\infty} G_{m}\left(\rho, \rho_{0}\right) \cos \left[m\left(\varphi-\varphi_{0}\right)\right],
$$

where $G_{m}\left(\rho, \rho_{0}\right)$ is a Green function of the following boundary-value problem

$$
\left\{\begin{array}{l}
L_{m} G_{m}:=\frac{1}{\rho} \frac{d}{d \rho}\left(\rho \frac{d G_{m}}{d \rho}\right)-\left(\frac{m^{2}}{\rho^{2}}+\kappa_{2}^{2}\right) G_{m}=-\frac{\delta\left(\rho-\rho_{0}\right)}{2 \pi \rho}, \rho_{0} \geq r \\
G_{m}\left(r, \rho_{0}\right)=0, \\
G_{m}\left(\rho, \rho_{0}\right)=O(1 / \rho), \rho \rightarrow \infty, \text { uniformly w.r.t } m .
\end{array}\right.
$$


It is easy to verify that the Green's function $G_{m}$ has the form

$$
G_{m}\left(\rho, \rho_{0}\right)=\left\{\begin{array}{l}
\frac{K_{m}\left(\kappa_{2} \rho_{0}\right)}{K_{m}\left(\kappa_{2} r\right)}\left(I_{m}\left(\kappa_{2} r\right) K_{m}\left(\kappa_{2} \rho\right)-I_{m}\left(\kappa_{2} \rho\right) K_{m}\left(\kappa_{2} r\right)\right), r \leq \rho<\rho_{0} \\
\frac{K_{m}\left(\kappa_{2} \rho\right)}{K_{m}\left(\kappa_{2} r\right)}\left(I_{m}\left(\kappa_{2} \rho_{0}\right) K_{m}\left(\kappa_{2} r\right)-I_{m}\left(\kappa_{2} r\right) K_{m}\left(\kappa_{2} \rho_{0}\right)\right), r \leq \rho_{0}<\rho,
\end{array}\right.
$$

where $I_{m}$ and $K_{m}$ are the modified Bessel functions [29].

Finally we obtain

$$
G\left(x, x_{0}\right)=\frac{1}{2 \pi} \begin{cases}\sum_{m=-\infty}^{\infty} \frac{K_{m}\left(\kappa_{2} \rho_{0}\right)}{K_{m}\left(\kappa_{2} r\right)} \xi_{1} \cos \left[m\left(\varphi-\varphi_{0}\right)\right], & r \leq \rho<\rho_{0} \\ \sum_{m=-\infty}^{\infty} \frac{K_{m}\left(\kappa_{2} \rho\right)}{K_{m}\left(\kappa_{2} r\right)} \xi_{2} \cos \left[m\left(\varphi-\varphi_{0}\right)\right], & r \leq \rho_{0}<\rho\end{cases}
$$

where

$$
\begin{aligned}
& \xi_{1}:=I_{m}\left(\kappa_{2} r\right) K_{m}\left(\kappa_{2} \rho\right)-I_{m}\left(\kappa_{2} \rho\right) K_{m}\left(\kappa_{2} r\right), \\
& \xi_{2}:=I_{m}\left(\kappa_{2} \rho_{0}\right) K_{m}\left(\kappa_{2} r\right)-I_{m}\left(\kappa_{2} r\right) K_{m}\left(\kappa_{2} \rho_{0}\right) .
\end{aligned}
$$

Using the second Green's formula, we have

$$
\int_{\mathbb{R}^{2} \backslash B_{r}}(u L v-v L u) d x=\left.r \int_{0}^{2 \pi}\left(u \frac{\partial v}{\partial \rho}-v \frac{\partial u}{\partial \rho}\right)\right|_{\rho=r} d \varphi .
$$

Next, let $v=G$, then we get

$$
\int_{\mathbb{R}^{2} \backslash B_{r}}(u L G-G L u) d x=\left.r \int_{0}^{2 \pi} u \frac{\partial G}{\partial \rho}\right|_{\rho=r} d \varphi .
$$

Moreover

$$
\int_{\mathbb{R}^{2} \backslash B_{r}}(u L G-G L u) d x=-u\left(x_{0}\right), x_{0} \in \mathbb{R}^{2} \backslash \bar{B}_{r} .
$$

Finally we obtain

$$
u\left(x_{0}\right)=-\left.r \int_{0}^{2 \pi} u(x) \frac{\partial G\left(x, x_{0}\right)}{\partial \rho}\right|_{\rho=r} d \varphi, x_{0} \in \mathbb{R}^{2} \backslash \bar{B}_{r},
$$

where

$$
\left.\frac{\partial G\left(x, x_{0}\right)}{\partial \rho}\right|_{\rho=r}=-\frac{1}{2 \pi r} \sum_{m=-\infty}^{\infty} \frac{K_{m}\left(\kappa_{2} \rho_{0}\right)}{K_{m}\left(\kappa_{2} r\right)} \cos \left[m\left(\varphi-\varphi_{0}\right)\right]
$$

Taking into account the theorem on the continuity of the normal derivative of the double layer potential on the boundary [6] and using (3.4), we define the value of the normal derivatives on the boundary $\Gamma_{2}$

$$
\left.\frac{\partial u}{\partial \rho_{0}}\right|_{\rho_{0}=r}=-\left.r \int_{0}^{2 \pi} u(x) \frac{\partial^{2} G\left(x, x_{0}\right)}{\partial \rho_{0} \partial \rho}\right|_{\substack{\rho=r \\ \rho_{0}=r}} d \varphi
$$


and

$$
\left.\frac{\partial^{2} G\left(x, x_{0}\right)}{\partial \rho_{0} \partial \rho}\right|_{\substack{\rho=r \\ \rho=r}}=-\frac{\kappa_{2}}{2 \pi r} \sum_{m=-\infty}^{\infty} \frac{K_{m}^{\prime}\left(\kappa_{2} r\right)}{K_{m}\left(\kappa_{2} r\right)} \cos \left[m\left(\varphi-\varphi_{0}\right)\right] .
$$

The convergence of the series (3.5) and (3.6) is understood in the sense of distributions [10].

Further, rearranging the variables $x \leftrightarrow x_{0}$, multiplying by the test functions $u$ and $v$ and taking the integrals $\int_{\Gamma_{2}} \frac{\partial u}{\partial \rho} \bar{v} d l$ we get

$$
\begin{gathered}
\int_{\Gamma_{2}} \frac{\partial \Pi}{\partial \rho} \bar{u} d l=-\left.r^{2} \int_{0}^{2 \pi} \int_{0}^{2 \pi} \frac{\partial^{2} G\left(x, x_{0}\right)}{\partial \rho \partial \rho_{0}} \Pi\left(x_{0}\right) \bar{u}(x)\right|_{\substack{\rho=r \\
\rho=r}} d \varphi d \varphi_{0}, \\
\int_{\Gamma_{2}} \frac{\partial \Phi}{\partial \rho} \bar{v} d l=-\left.r^{2} \int_{0}^{2 \pi} \int_{0}^{2 \pi} \frac{\partial^{2} G\left(x, x_{0}\right)}{\partial \rho \partial \rho_{0}} \Phi\left(x_{0}\right) \bar{v}(x)\right|_{\substack{\rho=r \\
\rho=r}} ^{\substack{\rho=r \\
0}} d \varphi d \varphi_{0} .
\end{gathered}
$$

Applying the transmission conditions (2.10) we obtain

$$
\begin{aligned}
& -\left.r^{2} \int_{0}^{2 \pi} \int_{0}^{2 \pi} \frac{\partial^{2} G\left(x, x_{0}\right)}{\partial \rho \partial \rho_{0}} \Pi\left(x_{0}\right) \bar{u}(x)\right|_{\substack{\rho=r \\
\rho_{0}=r}} d \varphi d \varphi_{0} \\
& \quad=-\gamma \int_{\Gamma_{1}}\left(\frac{\kappa_{2}^{2}}{\kappa_{1}^{2}} \frac{\partial \Phi}{\partial \tau}+\frac{\partial \Phi}{\partial \tau}\right) \bar{u} d \tau-\kappa_{2}^{2} \int_{\Omega} \frac{\widetilde{\varepsilon}}{\tilde{k}^{2}} \nabla \Pi \nabla \bar{u} d x+\kappa_{2}^{2} \int_{\Omega} \widetilde{\varepsilon} \Pi \bar{u} d x \\
& -\left.r^{2} \int_{0}^{2 \pi} \int_{0}^{2 \pi} \frac{\partial^{2} G\left(x, x_{0}\right)}{\partial \rho \partial \rho_{0}} \Phi\left(x_{0}\right) \bar{v}(x)\right|_{\substack{\rho=r \\
\rho_{0}=r}} d \varphi d \varphi_{0} \\
& =\gamma \int_{\Gamma_{1}}\left(\frac{\kappa_{2}^{2}}{\kappa_{1}^{2}} \frac{\partial \Pi}{\partial \tau}+\frac{\partial \Pi}{\partial \tau}\right) \bar{v} d \tau-\kappa_{2}^{2} \int_{\Omega} \frac{1}{\tilde{k}^{2}} \nabla \Phi \nabla \bar{v} d x+\kappa_{2}^{2} \int_{\Omega} \Phi \bar{v} d x
\end{aligned}
$$

Summing up the last expressions, we get

$$
\begin{aligned}
\kappa_{2}^{2} \int_{\Omega} \frac{1}{\tilde{k}^{2}}(\widetilde{\varepsilon} \nabla \Pi \nabla \bar{u}+\nabla \Phi \nabla \bar{v}) d x-\kappa_{2}^{2} \int_{\Omega}(\widetilde{\varepsilon} \Pi \bar{u}+\Phi \bar{v}) d x \\
+\gamma\left(\frac{\kappa_{2}^{2}}{\kappa_{1}^{2}}+1\right) \int_{\Gamma_{1}}\left(\frac{\partial \Pi}{\partial \tau} \bar{v}-\frac{\partial \Phi}{\partial \tau} \bar{u}\right) d \tau \\
-\left.r^{2} \int_{0}^{2 \pi} \int_{0}^{2 \pi} \frac{\partial^{2} G\left(x, x_{0}\right)}{\partial \rho \partial \rho_{0}}\left(\Pi\left(x_{0}\right) \bar{u}(x)+\Phi\left(x_{0}\right) \bar{v}(x)\right)\right|_{\substack{\rho=r \\
\rho_{0}=r}} d \varphi d \varphi_{0}=0 .
\end{aligned}
$$

Multiplying the last relation by $\gamma^{2}$, we obtain the variational relation

$$
\begin{aligned}
& \gamma^{4} \int_{\Omega}(\widetilde{\varepsilon} \Pi \bar{u}+\Phi \bar{v}) d x+\gamma^{2}\left(\int_{\Omega}(\widetilde{\varepsilon} \nabla \Pi \nabla \bar{u}+\nabla \Phi \nabla \bar{v}) d x\right. \\
& \left.-\left(\varepsilon_{1}+\varepsilon_{2}\right) \int_{\Omega}(\widetilde{\varepsilon} \Pi \bar{u}+\Phi \bar{v}) d x\right)+\gamma\left(\varepsilon_{1}-\varepsilon_{2}\right) \int_{\Gamma_{1}}\left(\frac{\partial \Pi}{\partial \tau} \bar{v}-\frac{\partial \Phi}{\partial \tau} \bar{u}\right) d \tau \\
& +\varepsilon_{1} \varepsilon_{2}\left(\int_{\Omega}(\widetilde{\varepsilon} \Pi \bar{u}+\Phi \bar{v}) d x-\int_{\Omega}\left(\nabla \Pi \nabla \bar{u}+\frac{1}{\widetilde{\varepsilon}} \nabla \Phi \nabla \bar{v}\right) d x\right) \\
& -\left.r^{2} \kappa_{1}^{2} \int 0^{2 \pi} \int_{0}^{2 \pi} \frac{\partial^{2} G\left(x, x_{0}\right)}{\partial \rho \partial \rho_{0}}\left(\Pi\left(x_{0}\right) \bar{u}(x)+\Phi\left(x_{0}\right) \bar{v}(x)\right)\right|_{\substack{\rho=r \\
\rho=0}} d \varphi d \varphi_{0}=0 .
\end{aligned}
$$


Definition 1. The pair of functions

$$
\Pi \in H_{0}^{1}(\Omega), \Phi \in H^{1}(\Omega),\left(\|\Pi\|_{1}+\|\Phi\|_{1} \neq 0\right)
$$

is called the eigenvector of the problem (2.6)-(2.11) corresponding to the eigenvalue $\gamma_{0} \in \mathbb{C}$ if the variational relation (3.7) holds for $u \in H_{0}^{1}(\Omega), v \in H^{1}(\Omega)$.

\section{The problem of the spectrum of the operator-function}

Let $H=H_{0}^{1}(\Omega) \times H^{1}(\Omega)$ be the Cartesian product of the Hilbert spaces with the inner product and the norm

$$
(\mathbf{u}, \mathbf{v})=\left(u_{1}, v_{1}\right)_{1}+\left(u_{2}, v_{2}\right)_{1},\|\mathbf{u}\|^{2}=\left\|u_{1}\right\|_{1}^{2}+\left\|u_{2}\right\|_{1}^{2} ; \mathbf{u}, \mathbf{v} \in H,
$$

where

$$
\mathbf{u}=\left(u_{1}, u_{2}\right)^{T}, \mathbf{v}=\left(v_{1}, v_{2}\right)^{T}, u_{1}, v_{1} \in H_{0}^{1}(\Omega), u_{2}, v_{2} \in H^{1}(\Omega) .
$$

The integrals in (3.7) can be considered as the sesquilinear forms defined in $H$ with respect to vector-functions

$$
\mathbf{u}=(\Pi, \Phi)^{T}, \mathbf{v}=(u, v)^{T} .
$$

These forms (if they are bounded) define, in accordance with the results of [8], linear bounded operators $\mathrm{T}: H \rightarrow H$

$$
\mathrm{t}(\mathbf{u}, \mathbf{v})=(\mathrm{T} \mathbf{u}, \mathbf{v}), \forall \mathbf{v} \in H
$$

Linearity results from the linear property of the form with respect to the first argument and the continuity property follows from the estimates

$$
\|\mathrm{T} \mathbf{u}\|^{2}=\mathrm{t}(\mathbf{u}, \mathrm{T} \mathbf{u}) \leq C\|\mathbf{u}\|\|\mathrm{T} \mathbf{u}\| .
$$

Let us consider the following forms and corresponding operators

$$
\begin{aligned}
& \mathrm{k}(\mathbf{u}, \mathbf{v}):=\int_{\Omega}(\widetilde{\varepsilon} \Pi \bar{u}+\Phi \bar{v}) d x=(\mathrm{K} \mathbf{u}, \mathbf{v}), \forall \mathbf{v} \in H, \\
& \widetilde{\mathrm{k}}(\mathbf{u}, \mathbf{v}):=\int_{\Omega} \frac{\widetilde{\varepsilon}+1}{\widetilde{\varepsilon}}(\widetilde{\varepsilon} \Pi \bar{u}+\Phi \bar{v}) d x=(\widetilde{\mathrm{K}} \mathbf{u}, \mathbf{v}), \forall \mathbf{v} \in H, \\
& \mathrm{a}(\mathbf{u}, \mathbf{v}):=\int_{\Omega}(\widetilde{\varepsilon} \nabla \Pi \nabla \bar{u}+\nabla \Phi \nabla \bar{v}+\widetilde{\varepsilon} \Pi \bar{u}+\Phi \bar{v}) d x=(\mathrm{Au}, \mathbf{v}), \forall \mathbf{v} \in H, \\
& \widetilde{\mathrm{a}}(\mathbf{u}, \mathbf{v}):=\int_{\Omega}\left(\nabla \Pi \nabla \bar{u}+\frac{1}{\widetilde{\varepsilon}} \nabla \Phi \nabla \bar{v}+\Pi \bar{u}+\frac{1}{\widetilde{\varepsilon}} \Phi \bar{v}\right) d x=(\widetilde{\mathrm{A}} \mathbf{u}, \mathbf{v}), \forall \mathbf{v} \in H, \\
& \mathrm{~s}(\mathbf{u}, \mathbf{v}):=\int_{\Gamma_{1}}\left(\frac{\partial \Pi}{\partial \tau} \bar{v}-\frac{\partial \Phi}{\partial \tau} \bar{u}\right) d \tau=(\mathrm{Su}, \mathbf{v}), \forall \mathbf{v} \in H, \\
& \mathrm{p}(\mathbf{u}, \mathbf{v}):=\left.\int_{0}^{2 \pi} \int_{0}^{2 \pi} F\left(\varphi, \varphi_{0}\right)\left(\Pi\left(x_{0}\right) \bar{u}(x)+\Phi\left(x_{0}\right) \bar{v}(x)\right)\right|_{\substack{\rho=r \\
\rho_{0}=r}} d \varphi d \varphi_{0} \\
&=(\mathrm{P} \mathbf{u}, \mathbf{v}), \forall \mathbf{v} \in H,
\end{aligned}
$$




$$
F\left(\varphi, \varphi_{0}\right):=-\left.r^{2} \kappa_{1}^{2} \frac{\partial^{2} G\left(x, x_{0}\right)}{\partial \rho \partial \rho_{0}}\right|_{\substack{\rho=r \\ \rho_{0}=r}} .
$$

It is known that the Green's function $G\left(x, x_{0}\right)$ has a logarithmic singularity that can be isolated. Then, as in [16] we find

$$
\begin{gathered}
\left.\frac{\partial^{2} G\left(x, x_{0}\right)}{\partial \rho_{0} \partial \rho}\right|_{\substack{\rho=r \\
\rho_{0}=r}}=-\frac{\kappa_{2}}{2 \pi r} \sum_{m=-\infty}^{\infty} \frac{K_{m}^{\prime}\left(\kappa_{2} r\right)}{K_{m}\left(\kappa_{2} r\right)} \cos \left[m\left(\varphi-\varphi_{0}\right)\right] \\
=\frac{\kappa_{2}}{\pi r} \frac{K_{1}\left(\kappa_{2} r\right)}{K_{0}\left(\kappa_{2} r\right)}-\frac{\kappa_{2}}{\pi r} \sum_{m=1}^{\infty} \frac{K_{m}^{\prime}\left(\kappa_{2} r\right)}{K_{m}\left(\kappa_{2} r\right)} \cos \left[m\left(\varphi-\varphi_{0}\right)\right] \\
=\frac{\kappa_{2}}{\pi r} \frac{K_{1}\left(\kappa_{2} r\right)}{K_{0}\left(\kappa_{2} r\right)}-\frac{1}{\pi r^{2}} \sum_{m=1}^{\infty}\left(\kappa_{2} r \frac{K_{m}^{\prime}\left(\kappa_{2} r\right)}{K_{m}\left(\kappa_{2} r\right)}+m\right) \cos \left[m\left(\varphi-\varphi_{0}\right)\right] \\
\quad+\frac{1}{\pi r^{2}} \sum_{m=1}^{\infty} m \cos \left[m\left(\varphi-\varphi_{0}\right)\right] \\
=\frac{\kappa_{2}}{\pi r} \frac{K_{1}\left(\kappa_{2} r\right)}{K_{0}\left(\kappa_{2} r\right)}-\frac{1}{\pi r^{2}} \sum_{m=1}^{\infty}\left(\kappa_{2} r \frac{K_{m}^{\prime}\left(\kappa_{2} r\right)}{K_{m}\left(\kappa_{2} r\right)}+m\right) \cos \left[m\left(\varphi-\varphi_{0}\right)\right] \\
-\frac{1}{\pi r^{2}} \frac{1}{1-\cos \left(\varphi-\varphi_{0}\right)} .
\end{gathered}
$$

We have $\kappa_{2} r \frac{K_{m}^{\prime}\left(\kappa_{2} r\right)}{K_{m}\left(\kappa_{2} r\right)}+m=O\left(\frac{1}{m}\right)$ for $m \rightarrow \infty$, and the series converges conditionally for $\varphi \neq \varphi_{0}$. We represent the operator $\mathrm{P}(\gamma)$ as the sum of a compact and a hypersingular operator

$$
\mathrm{P}(\gamma)=\mathrm{K}_{\mathrm{P}}(\gamma)+\left(\gamma^{2}-\varepsilon_{1}\right) \widetilde{\mathrm{P}}
$$

where

$$
\begin{aligned}
& \widetilde{\mathrm{p}}(\mathbf{u}, \mathbf{v})=-\left.\frac{1}{2 \pi} \int_{0}^{2 \pi} \int_{0}^{2 \pi} \frac{\Pi\left(x_{0}\right) \bar{u}(x)+\Phi\left(x_{0}\right) \bar{v}(x)}{1-\cos \left(\varphi-\varphi_{0}\right)}\right|_{\substack{\rho=r \\
\rho=r}} d \varphi d \varphi_{0}=(\widetilde{\mathrm{P}} \mathbf{u}, \mathbf{v}), \forall \mathbf{v} \in H, \\
& \kappa_{\mathrm{p}}(\mathbf{u}, \mathbf{v})=-\left.\frac{\kappa_{1}^{2} \kappa_{2} r}{\pi} \frac{K_{1}\left(\kappa_{2} r\right)}{K_{0}\left(\kappa_{2} r\right)} \int_{0}^{2 \pi} \int_{0}^{2 \pi}\left(\Pi\left(x_{0}\right) \bar{u}(x)+\Phi\left(x_{0}\right) \bar{v}(x)\right)\right|_{\substack{\rho=r \\
\rho_{0}=r}} d \varphi d \varphi_{0} \\
& \quad+\frac{\kappa_{1}^{2}}{\pi} \int_{0}^{2 \pi} \int_{0}^{2 \pi} \sum_{m=1}^{\infty}\left(\kappa_{2} r \frac{K_{m}^{\prime}\left(\kappa_{2} r\right)}{K_{m}\left(\kappa_{2} r\right)}+m\right) \cos \left[m\left(\varphi-\varphi_{0}\right)\right] \\
& \quad \times\left.\left(\Pi\left(x_{0}\right) \bar{u}(x)+\Phi\left(x_{0}\right) \bar{v}(x)\right)\right|_{\substack{\rho=r \\
\rho_{0}=r}} d \varphi d \varphi_{0}=\left(\mathrm{K}_{\mathrm{P}}(\gamma) \mathbf{u}, \mathbf{v}\right), \forall \mathbf{v} \in H .
\end{aligned}
$$

Since $\Pi, u, \Phi, v \in H^{1}(\Omega)$, then the traces of these functions on $\Gamma_{2}$ belong to the space $H^{1 / 2}\left(\Gamma_{2}\right)[2]$. The hypersingular operator $\widetilde{\mathrm{P}}: H^{1 / 2}\left(\Gamma_{2}\right) \rightarrow$ $H^{-1 / 2}\left(\Gamma_{2}\right)$ is bounded [7]. Taking into account the antidual pairing of the spaces $H^{1 / 2}\left(\Gamma_{2}\right)$ and $H^{-1 / 2}\left(\Gamma_{2}\right)$ [27], we get that the sesquilinear form $\widetilde{\mathrm{p}}(\mathbf{u}, \mathbf{v})$ is bounded in $H$. Further, taking into account the rate of decrease of the coefficients of the series for $\kappa_{\mathrm{p}}(\mathbf{u}, \mathbf{v})$, we find [16] that this series will define an integral operator with a logarithmic singularity of the kernel, and the operator $\widetilde{\mathrm{K}}_{\mathrm{P}}: H^{1 / 2}\left(\Gamma_{2}\right) \rightarrow H^{-1 / 2}\left(\Gamma_{2}\right)$ will be compact. Thus, $P(\gamma): H \rightarrow H$ is bounded. The boundedness of the first five sesquilinear forms is proved in [19]. 
Now the variational problem (3.7) can be written in the operator form

$$
(\mathrm{N}(\gamma) \mathbf{u}, \mathbf{v})=0, \forall \mathbf{u} \in H
$$

or, equivalently,

$$
\begin{aligned}
\mathrm{N}(\gamma) \mathbf{u} & =0, \mathrm{~N}(\gamma): H \rightarrow H, \quad \mathrm{~N}(\gamma):=\gamma^{4} \mathrm{~K}+\gamma^{2}\left(\mathrm{~A}-\left(\varepsilon_{1}+\varepsilon_{2}+1\right) \mathrm{K}\right) \\
& +\gamma\left(\varepsilon_{1}-\varepsilon_{2}\right) \mathrm{S}-\varepsilon_{1} \varepsilon_{2}(\widetilde{\mathrm{A}}-\widetilde{\mathrm{K}})+\mathrm{P}(\gamma)
\end{aligned}
$$

Equation (4.1) is the operator form of the variational relation (3.7). The characteristic numbers and eigenvectors of the operator-function $\mathrm{N}(\gamma)$ by definition coincide with the eigenvalues and eigenvectors of the problem (2.6)-(2.11) for $\gamma^{2} \neq \widetilde{\varepsilon}$. Thus, the problem of normal waves is reduced to the eigenvalue problem for the operator-function $\mathrm{N}(\gamma)$.

\section{The properties of operator-function $\mathrm{N}(\gamma)$}

We give the following statements about the properties of operators occurring in $\mathrm{N}(\gamma)$ (proof in [19]):

Lemma 1. The operator $\mathrm{K}$ is positive, $\mathrm{K}>0$, and compact. The following estimate holds for its eigenvalues

$$
\lambda_{n}(\mathrm{~K})=O\left(n^{-1}\right), \quad n \rightarrow \infty .
$$

Lemma 2. The operators A, $\widetilde{\mathrm{A}}$ are uniformly positive:

$$
I \leq \mathrm{A} \leq \varepsilon_{2} I, \quad 1 / \varepsilon_{1} \mathrm{I} \leq \widetilde{\mathrm{A}} \leq \mathrm{I},
$$

where $I$ is the unit operator in $H$.

Lemma 3. The operator $\mathrm{S}$ is self-adjoint, $\mathrm{S}=\mathrm{S}^{*}$, and the following inequalities hold

$$
-\frac{1}{2} I \leq \mathrm{S} \leq \frac{1}{2} I
$$

A special attention is required to consider the properties of the trace operator P. In [7] this operator is studied in detail as a pseudodifferential operator in Sobolev spaces.

Lemma 4. The operator $\mathrm{P}$ is a self-adjoint bounded operator.

DEFINITION 2. We will denote by $\rho(\mathrm{N})$ the resolvent set of $\mathrm{N}(\gamma)$ (consisting of all values of $\gamma \in \mathbb{C}$ where there exists the bounded inverse operator $\mathrm{N}^{-1}(\gamma)$ ) and by $\sigma(\mathrm{N})=\mathbb{C} \backslash \rho(\mathrm{N})$ the spectrum of $\mathrm{N}(\gamma)$.

Properties of the spectrum of the operator-function $\mathrm{N}(\gamma)$ are given in the following theorems.

Theorem 1. There exists $\widetilde{\gamma} \in \mathbb{R}$ such that the operator $\mathrm{N}(\widetilde{\gamma})$ is continuously invertible, i.e. the resolvent set $\varrho(\mathrm{N}):=\left\{\gamma: \exists \mathrm{N}^{-1}(\gamma): H \rightarrow H\right\}$ of operatorfunction $\mathrm{N}(\widetilde{\gamma})$ is not empty. 
Proof. Let $\gamma \in \mathbb{R}$ and $\gamma \rightarrow+\infty$. Consider the following operator-function $\frac{1}{\gamma^{2}} \mathrm{~N}(\gamma)=\mathrm{N}_{1}(\gamma)+\mathrm{N}_{2}(\gamma)$, where

$$
\begin{aligned}
& \mathrm{N}_{1}(\gamma)=\gamma^{2} \mathrm{~K}+\mathrm{A}-\left(\varepsilon_{1}+\varepsilon_{2}+1\right) \mathrm{K}+\gamma \mathrm{P}_{0}, \\
& \mathrm{~N}_{2}(\gamma)=\frac{\varepsilon_{1}-\varepsilon_{2}}{\gamma} \mathrm{S}-\frac{\varepsilon_{1} \varepsilon_{2}}{\gamma^{2}}(\widetilde{\mathrm{A}}-\widetilde{\mathrm{K}})+\frac{1}{\gamma^{2}} \mathrm{P}(\gamma)-\gamma \mathrm{P}_{0},
\end{aligned}
$$

and the operator $\mathrm{P}_{0}: H \rightarrow H$ is defined by a sesquilinear form

$$
\begin{aligned}
& \mathrm{p}_{0}(\mathbf{u}, \mathbf{v}):=\int_{0}^{2 \pi} \sum_{m=-\infty}^{\infty} \cos \left[m\left(\varphi-\varphi_{0}\right)\right] d \varphi \\
& \quad \times\left.\int_{0}^{2 \pi}\left(\Pi\left(x_{0}\right) \bar{u}(x)+\Phi\left(x_{0}\right) \bar{v}(x)\right)\right|_{\substack{\rho=r \\
\rho_{0}=r}} d \varphi_{0}=\left(\mathrm{P}_{0} \mathbf{u}, \mathbf{v}\right), \forall \mathbf{v} \in H .
\end{aligned}
$$

It is known [10] that (in the sense of distributions)

$$
\sum_{m=-\infty}^{\infty} \cos m \phi=2 \pi \sum_{l=-\infty}^{\infty} \delta(\phi-2 \pi l)=2 \pi \delta(\phi)
$$

where $\phi=\left|\varphi-\varphi_{0}\right|, 0 \leq|\phi| \leq \pi$. We get that

$$
\sum_{m=-\infty}^{\infty} \cos \left[m\left(\varphi-\varphi_{0}\right)\right]=2 \pi \delta\left(\varphi-\varphi_{0}\right) .
$$

Then the operator $\mathrm{P}_{0}$ takes the form

$$
\left(\mathrm{P}_{0} \mathbf{u}, \mathbf{v}\right):=\left.2 \pi \int_{0}^{2 \pi}(\Pi(x) \bar{u}(x)+\Phi(x) \bar{v}(x))\right|_{\rho=r} d \varphi, \forall \mathbf{v} \in H .
$$

It is obvious that

$$
\left(\mathrm{P}_{0} \mathbf{u}, \mathbf{u}\right)=\left.2 \pi \int_{0}^{2 \pi}\left(|\Pi(x)|^{2}+|\Phi(x)|^{2}\right)\right|_{\rho=r} d \varphi>0 \text { for }\|\mathbf{u}\| \neq 0 .
$$

Since the traces of functions $\Pi, u, \Phi, v$ on $\Gamma_{2}$ belong to the space $H^{1 / 2}\left(\Gamma_{2}\right)$ we conclude that $P_{0}: H \rightarrow H$ is bounded.

Taking into account the asymptotic of the function $K_{m}$ for $\gamma \rightarrow+\infty$ [1], we have:

$$
\frac{1}{\gamma^{2}} F\left(\varphi, \varphi_{0}\right) \sim \gamma+O\left(\frac{1}{\gamma}\right)
$$

and hence

$$
\left\|\frac{1}{\gamma^{2}} \mathrm{P}(\gamma)-\gamma \mathrm{P}_{0}\right\|=O\left(\frac{1}{\gamma}\right), \text { for } \gamma \rightarrow+\infty .
$$

Then the operator-function $\gamma^{-2} \mathrm{~N}(\gamma)$ can be considered as a perturbation of the operator pencil $\mathrm{N}_{1}$ by the operator-function $\mathrm{N}_{2}$ for large $\gamma$.

Taking into account the properties of the operators $\mathrm{K}, \mathrm{A}_{1}$, we find that there is a large $\widetilde{\gamma}>0$ such that

$\operatorname{Re}\left(\mathrm{N}_{1}(\widetilde{\gamma}) \mathbf{u}, \mathbf{u}\right)=\widetilde{\gamma}^{2}(\mathrm{~K} \mathbf{u}, \mathbf{u})+(\mathrm{Au}, \mathbf{u})-\left(\varepsilon_{1}+\varepsilon_{2}+1\right)(\mathrm{Ku}, \mathbf{u})+\widetilde{\gamma}\left(\mathrm{P}_{0} \mathbf{u}, \mathbf{u}\right) \geq\|\mathbf{u}\|^{2}$ 
for any $\mathbf{u}$. Hence $\widetilde{\gamma} \in \rho\left(\mathrm{N}_{1}\right)$, where $\varrho\left(\mathrm{N}_{1}\right)$ is the resolvent set of the operatorfunction $\mathrm{N}_{1}$. Moreover, theorem 4.1 in [5] gives the estimate

$$
\left\|\mathrm{N}_{1}^{-1}(\widetilde{\gamma})\right\| \leq 1
$$

We take $\widetilde{\gamma}$ such that the inequality $\left\|\mathrm{N}_{2}(\widetilde{\gamma})\right\|<1$ is satisfied as well and find that there exists a bounded operator

$$
\left(\mathrm{N}_{1}(\widetilde{\gamma})+\mathrm{N}_{2}(\widetilde{\gamma})\right)^{-1}=\left(\mathrm{I}+\mathrm{N}_{1}^{-1}(\widetilde{\gamma}) \mathrm{N}_{2}(\widetilde{\gamma})\right) \mathrm{N}_{1}^{-1}(\widetilde{\gamma})
$$

The proof of the theorem is complete.

Lemma 5. The operator $\widetilde{\mathrm{P}}$ is positive, $\widetilde{\mathrm{P}}>0$.

Proof. Consider the scalar product $(\widetilde{\mathrm{P}} \mathbf{u}, \mathbf{u})$. We represent the function $\Pi$ and $\Phi$ in the form of Fourier series

$$
\begin{aligned}
& \Pi(\varphi)=\sum_{n=-\infty}^{\infty} X_{n} e^{i n \varphi}, \bar{\Pi}\left(\varphi_{0}\right)=\sum_{m=-\infty}^{\infty} \bar{X}_{m} e^{-i m \varphi_{0}}, \\
& \Phi(\varphi)=\sum_{p=-\infty}^{\infty} Y_{p} e^{i p \varphi}, \bar{\Phi}\left(\varphi_{0}\right)=\sum_{q=-\infty}^{\infty} \bar{Y}_{q} e^{-i q \varphi_{0}},
\end{aligned}
$$

and using the technique of distribution [28], the quadratic form takes the form $(\|\mathbf{u}\| \neq 0)$ :

$$
\begin{aligned}
(\widetilde{\mathrm{P}} \mathbf{u}, \mathbf{u}) & =-\left.\frac{1}{2 \pi} \int_{0}^{2 \pi} \int_{0}^{2 \pi} \frac{\Pi\left(x_{0}\right) \bar{\Pi}(x)+\Phi\left(x_{0}\right) \bar{\Phi}(x)}{1-\cos \left(\varphi-\varphi_{0}\right)}\right|_{\substack{\rho=r \\
\rho=r}} d \varphi d \varphi_{0} \\
& =\sum_{j=1}^{\infty} 2 \pi j\left(\left|X_{j}\right|^{2}+\left|Y_{j}\right|^{2}\right)>0 .
\end{aligned}
$$

We can rewrite the operator-function $\mathrm{N}(\gamma)$ as follows $\mathrm{N}(\gamma):=\mathrm{N}_{\mathrm{K}}(\gamma)+\mathrm{N}_{0}(\gamma)$, where

$$
\begin{aligned}
& \mathrm{N}_{\mathrm{K}}(\gamma)=\gamma^{4} \mathrm{~K}-\gamma^{2}\left(\varepsilon_{1}+\varepsilon_{2}+1\right) \mathrm{K}+\varepsilon_{1} \varepsilon_{2} \widetilde{\mathrm{K}}+\mathrm{K}_{\mathrm{P}}(\gamma), \\
& \mathrm{N}_{0}(\gamma)=\gamma^{2}(\mathrm{~A}+\widetilde{\mathrm{P}})+\gamma\left(\varepsilon_{1}-\varepsilon_{2}\right) \mathrm{S}-\varepsilon_{1}\left(\varepsilon_{2} \widetilde{\mathrm{A}}+\widetilde{\mathrm{P}}\right) .
\end{aligned}
$$

Theorem 2. The operator $\mathrm{N}(\gamma): H \rightarrow H$ is bounded, holomorphic, and Fredholm in the domain

$$
\Lambda=\mathbb{C} \backslash \widetilde{\Lambda} \text { and } \tilde{\Lambda}:=\left\{\gamma: \operatorname{Im} \gamma^{2}=0, \gamma^{2} \leq \varepsilon_{2}\right\} \cup\left\{\gamma: \operatorname{Im} \gamma=0, \gamma_{*} \leq|\gamma| \leq \gamma^{*}\right\}
$$

where

$$
\begin{aligned}
& \gamma_{*}:=\frac{-\epsilon+\sqrt{\epsilon^{2}+4 \varepsilon_{2}}}{2\left(\varepsilon_{1}+\|\widetilde{\mathrm{P}}\|\right)}, \quad \gamma^{*}:=\frac{\epsilon+\sqrt{\epsilon^{2}+4 \varepsilon_{1}\left(\varepsilon_{1}+\|\widetilde{\mathrm{P}}\|\right)\left(\varepsilon_{2}+\|\widetilde{\mathrm{P}}\|\right)}}{2}, \\
& \epsilon=0.5 *\left(\varepsilon_{1}-\varepsilon_{2}\right), \quad\left(\gamma_{*}<\sqrt{\varepsilon_{2}}, \gamma^{*}>\sqrt{\varepsilon_{1}}\right) .
\end{aligned}
$$


Proof. In the domain $\left\{\gamma: \operatorname{Im} \gamma^{2}=0, \gamma^{2} \leq \varepsilon_{2}\right\}$ the functions

$$
\kappa_{2} \frac{K_{m}^{\prime}\left(\kappa_{2} r\right)}{K_{m}\left(\kappa_{2} r\right)}=-\kappa_{2} \frac{K_{m+1}\left(\kappa_{2} r\right)}{K_{m}\left(\kappa_{2} r\right)}+\frac{m}{r}
$$

are analytical (as a function of $\gamma$ ). Since $\operatorname{Re} \kappa_{2}>0$, the functions $K_{m}\left(\kappa_{2} r\right)$ have no zeros [1].

Let $\gamma=\gamma^{\prime}+i \gamma^{\prime \prime}, \gamma^{\prime \prime} \neq 0$. Then

$$
\frac{1}{\gamma^{\prime \prime}} \operatorname{Im}\left[\frac{\mathrm{N}_{0}(\gamma)}{\gamma}\right]=\mathrm{A}+\widetilde{\mathrm{P}}+\frac{\varepsilon_{1}}{|\gamma|^{2}}\left(\varepsilon_{2} \widetilde{\mathrm{A}}+\widetilde{\mathrm{P}}\right) \geq \mathrm{I} .
$$

Further, let $\operatorname{Im} \gamma=0$. Consider the following quadratic form

$$
\left(\mathrm{N}_{0}(\gamma) \mathbf{u}, \mathbf{u}\right)=\left(\left(\gamma^{2}(\mathrm{~A}+\widetilde{\mathrm{P}})+\gamma\left(\varepsilon_{1}-\varepsilon_{2}\right) \mathrm{S}-\varepsilon_{1}\left(\varepsilon_{2} \widetilde{\mathrm{A}}+\widetilde{\mathrm{P}}\right)\right) \mathbf{u}, \mathbf{u}\right)=0 .
$$

Expressing $(\mathrm{Su}, \mathbf{u})$ from the last equation and taking into account the estimate (5.2), we obtain the following double inequality

$$
-\epsilon \leq \frac{\varepsilon_{1}}{\gamma}\left(\varepsilon_{2}\|\widetilde{\mathrm{A}}\|+\|\widetilde{\mathrm{P}}\|\right)-\gamma(\|\mathrm{A}\|+\|\widetilde{\mathrm{P}}\|) \leq \epsilon .
$$

Solving the inequalities (5.3) with respect to $\gamma$ and taking into account the estimates (5.1), we find that outside the set $\gamma_{*} \leq|\gamma| \leq \gamma^{*}$ the operator $\mathrm{N}_{0}(\gamma)$ is positive or negative definite and therefore continuously invertible [5]. Hence the operator-function $\mathrm{N}(\gamma)$ is Fredholm as the sum of an invertible and compact operators, and ind $\mathrm{N}(\gamma)=0$.

The second assertion of the theorem follows from the fact that the variational relation (3.7) for $\gamma=\gamma_{2}$ or $\gamma=\gamma_{1}$ is identically equal to zero for functions $\Pi$ and $\Phi$, such that $\Pi, \Phi \in C_{0}^{\infty}\left(\bar{\Omega}_{*}\right)$, for $\bar{\Omega}_{*} \subset \Omega_{1}$ or $\bar{\Omega}_{*} \subset \Omega_{2}$, respectively.

Theorem 3. The spectrum of the operator-function $\mathrm{N}(\gamma): H \rightarrow H$ is discrete in $\Lambda$ i.e., it has a finite number of eigenvalues of finite algebraic multiplicity in any compact set $\mathrm{K}_{0} \subset \Lambda$. The points $\gamma_{j}= \pm \sqrt{\widetilde{\varepsilon}}$ denote the values of degeneration of the operator-function $\mathrm{N}(\gamma)$ where $\operatorname{dim} \operatorname{ker} \mathrm{N}\left(\gamma_{j}\right)=\infty$.

Proof. The assertion of the theorem is a corollary of theorem 2 and the theorem on the holomorphic operator-function [5]. The second assertion of the theorem follows from the fact that the variational relation (3.7) for $\gamma=\gamma_{2}$ or $\gamma=\gamma_{1}$ is identically equal to zero for functions $\Pi$ and $\Phi$, such that $\Pi, \Phi \in C_{0}^{\infty}\left(\bar{\Omega}_{*}\right)$, for $\bar{\Omega}_{*} \subset \Omega_{1}$ or $\bar{\Omega}_{*} \subset \Omega_{2}$, respectively.

Lemma 6. The spectrum of the operator-function $\mathrm{N}(\gamma)$ is symmetric with respect to the origin, $\sigma(\mathrm{N})=\sigma(-\mathrm{N})$. If $\gamma_{0}$ is an eigenvalue of the operatorfunction $\mathrm{N}(\gamma)$ corresponding to an eigenvector $\mathbf{u}=(\Pi, \Phi)^{T}$, then $-\gamma_{0}$ is also an eigenvalue of the operator-function $\mathrm{N}(\gamma)$ corresponding to an eigenvector $\mathbf{u}=(-\Pi, \Phi)^{T}$ with the same multiplicity. 


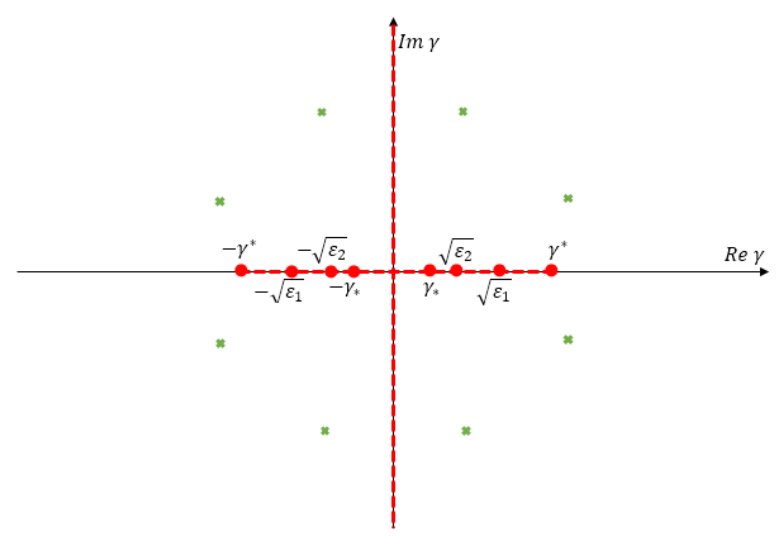

Figure 2. The spectrum of the operator-function $\mathrm{N}(\gamma) ; \times$ are eigenvalues of the operator-function $\mathrm{N}(\gamma)$; • denote values of degeneration of the operator-function $\mathrm{N}(\gamma)$; denote points of set $\widetilde{\Lambda}$.

Proof. Proof of the assertion is a simple verification of variational relation (3.7).

Figure 2 gives a visual representation of the distribution of the spectrum of the operator-function $\mathrm{N}(\gamma)$ on the complex plane. For points $\gamma \in \widetilde{\Lambda}$ an additional investigation is required.

\section{Conclusions}

We have reduced the boundary eigenvalue problem for the Maxwell equations describing normal waves in a broad class of non-homogeneously filled waveguides to an eigenvalue problem for an operator-function. We have proved fundamental properties of the spectrum of normal waves including the discreteness and a statement describing localization of eigenvalues of the operator-function on the complex plane.

It should be noted that the existence of propagating waves in open metaldielectric waveguides of arbitrary cross-section is not established.

\section{Acknowledgements}

The reported study was funded by RFBR, projects numbers 20-31-70010 and 19-31-51004.

\section{References}

[1] M. Abramowitz and I. Stegun. Handbook of Mathematical Functions with Formulas, Graphs, and Mathematical Tables. Dover Publications, 1965. https://doi.org/10.1063/1.3047921.

[2] R.A. Adams. Sobolev Spaces. Academic Press, 1975. 
[3] M. Costabel. Boundary integral operators on Lipschitz domains: Elementary results. SIAM J. Math. Anal., 19(3):613-626, 1988. https://doi.org/10.1137/0519043.

[4] A.L. Delitsyn. An approach to the completeness of normal waves in a waveguide with magnitodielectric filling. Differential Equations, 36:695-700, 2000. https://doi.org/10.1007/BF02754228.

[5] I.C Gohberg and M.G. Krein. Introduction to the Theory of Linear Nonselfadjoint Operators in Hilbert Space. Nauka, 1965.

[6] L.L. Helms. Introduction to potential theory. R. E. Krieger Pub. Co, 1975.

[7] A.S. Il'inskii and Yu.G. Smirnov. Diffraction of Electromagnetic Waves on Conductive Thin Screens: Pseudodifferential Operators in Diffraction Problems. Radiotehnika, 1996. (in Russian)

[8] T. Kato. Perturbation Theory for Linear Operators. New York: Springer-Verlag, 1980 .

[9] M.V. Keldysh. On the completeness of the eigenfunctions of some classes of non-selfadjoint linear operators. Doklady AN SSSR, 77:4-11, 1951.

[10] A.N Kolmogorov and S.V. Fomin. Elements of the Theory of Functions and Functional Analysis. Dover Publications, 1999.

[11] P.E. Krasnushkin and E.I. Moiseev. On the excitation of oscillations in layered radiowaveguide. Doklady AN SSSR, 264:1123-1127, 1982.

[12] L. Levin. Theory of waveguides. London: Newnes-Butterworths, 1975.

[13] V.V. Lozhechko and Yu.V. Shestopalov. Problems of the excitation of open cylindrical resonators with an irregular boundary. Computational Mathematics and Mathematical Physics, 35(1):53-61, 1995.

[14] Y. Shestopalov and Y. Smirnov. Eigenwaves in waveguides with dielectric inclusions: Completeness. Applicable Analysis, 93(9):1824-1845, 2014. https://doi.org/10.1080/00036811.2013.850494.

[15] Y. Shestopalov and Y. Smirnov. Eigenwaves in waveguides with dielectric inclusions: Spectrum. Applicable Analysis, 93(2):408-427, 2014. https://doi.org/10.1080/00036811.2013.778980.

[16] Yu.V. Shestopalov, Yu.G. Smirnov and E.V. Chernokozhin. Logarithmic Integral Equations in Electromagnetics. Holland: De Gruyter, 2000. https://doi.org/10.1515/9783110942057.

[17] Yu.G. Smirnov. Application of the operator pencil method in the eigenvalue problem for partially. Doklady AN SSSR, 312:597-599, 1990.

[18] Yu.G. Smirnov. The method of operator pencils in the boundary transmission problems for elliptic system of equations. Differ. Equ., 27:140-147, 1991.

[19] Yu.G. Smirnov. Mathematical Methods for Electromagnetic Problems. Penza: PSU Press, 2009.

[20] Yu.G. Smirnov and E. Smolkin. Discreteness of the spectrum in the problem on normal waves in an open inhomogeneous waveguide. Differential Equations, 53:1262-1273, 2017. https://doi.org/10.1134/S0012266117100032.

[21] Yu.G. Smirnov and E. Smolkin. Investigation of the spectrum of the problem of normal waves in a closed regular inhomogeneous dielectric waveguide of arbitrary cross section. Doklady Mathematics, 97(1):86-89, 2017. https://doi.org/10.1134/S1064562418010271. 
[22] Yu.G. Smirnov and E. Smolkin. Eigenwaves in a lossy metaldielectric waveguide. Applicable Analysis, 99(1):1-12, 2018. https://doi.org/10.1080/00036811.2018.1478084.

[23] Yu.G. Smirnov and E. Smolkin. Operator function method in the problem of normal waves in an inhomogeneous waveguide. Differential Equations, 54(9):11681179, 2018. https://doi.org/10.1134/S0012266118090057.

[24] Yu.G. Smirnov, E. Smolkin and M.O. Snegur. Analysis of the spectrum of azimuthally symmetric waves of an open inhomogeneous anisotropic waveguide with longitudinal magnetization. Computational Mathematics and Mathematical Physics, 58(11):1887-1901, 2018. https://doi.org/10.1134/S096554251811012X.

[25] E. Smolkin. Numerical method for electromagnetic wave propagation problem in a cylindrical inhomogeneous metal dielectric waveguiding structures. Mathematical Modelling and Analysis, 22(3):271-282, 2017. https://doi.org/10.3846/13926292.2017.1306809.

[26] A.W. Snyder and J. Love. Optical waveguide theory. Springer, 1983.

[27] H. Triebel. Theory of Function Spaces. Birkhauser Verlag, 1983. https://doi.org/10.1007/978-3-0346-0416-1.

[28] V.S. Vladimirov. Equations of Mathematical Physics. Nauka, 1985.

[29] G.N. Watson. A Treatise on the Theory of Bessel Functions. Cambridge University Press, 1995.

[30] A.S. Zilbergleit and Yu.I. Kopilevich. Spectral theory of guided waves. London: Inst. of Phys. Publ., 1966. 